\title{
Navigation network construction method for survey points using cartographic urban environment information
}

\author{
Ignat N. Penshin ${ }^{1, *}$, Larisa G. Evstratova ${ }^{1}$ \\ ${ }^{1}$ State University of Land Use Planning, 105064, 15 Kazakova str, Moscow, Russian Federation
}

\begin{abstract}
This research solves the problem of navigation network construction for panoramic survey points inside courtyards without using road network information. The described method of navigation network construction takes into account a number of geometric conditions that detecting and identifying the regularities of the decisive routing rules construction. An algorithm for constructing links between panoramic shooting points, based on the surrounding area and linear objects territory information, is presented. An intelligent agent of geographic information system has been developed that executes this task based on the proposed decision rules. The goals and objectives of the research are presented, input data array to solve the problem is considered, the formulation of geometric conditions, structure and object implementation of the algorithm are set out in this work. The task is viewed in terms of the perspective of graph theory, implemented in the DBMS PostgreSQL and illustrated in the QGIS geographic information system.
\end{abstract}

\section{Goals, objectives and subject of the research}

The active development of digital modeling of the urban environment poses new challenges to the developers of intelligent agents of geographic information systems. Currently, there is a sufficient abundance and variety of freely distributed spatial data arrays that display relevant environmental information. In this situation the construction of unique analytical models and derived data arrays that promote the consumer becomes the most demanded product.

The purpose of this research is to build a navigation network for a web service for viewing street panoramas. A distinctive feature of this service is the emphasis on intraquarterly shooting. This condition forms the task of building navigation within courtyards. A study of the quality and quantity of cartographic data on the urban road network showed a significant decrease useful information about roads inside courtyards. This conclusion is confirmed by the principle of classification of the road network, approved by the global Open Street Map (OSM) community, which prioritizes the road in the road network according to its connecting properties. Natural trails, footpaths, bicycle paths, and even

* Corresponding author: ignat.penshin51@gmail.com 
asphalt roads inside courtyards, due to the low priority of logistic importance, may have an unknown status or may not exist at all due to the lack of inspection or the inability to determine the relationship with the nearest roads in cameral conditions. In this situation, even with direct visibility between neighboring points of photographing, creating navigation between panoramic images based on road networks becomes difficult or impossible. Therefore, the classical method of using the road network to navigate through panoramic surveys becomes irrelevant; under these conditions, the developed intelligent agent solves this problem.

Further disclosure of the topic requires an unambiguous definition of the following concepts:

A spatial routing network is an object implementation of a graph in the form of a set of linear and point facilities. Adjacency and incidence of objects is expressed by the storage of attribute information by a point or linear network object.

Panoramic survey points - photographic points, the position information of which was obtained using a GNSS receiver. They are considered as graph vertices and are point objects with spatial coordinates on an ellipsoid (LBH).

The neighborhood of a given vertex is the set of all vertixes of the graph adjacent to this vertex through the incident edge.

A private graph is an initially undirected graph constructed for one initial vertex. A private graph defines a neighborhood of a given initial vertex. After constructing a private graph the edges can be represented as oriented. The concept was introduced to solve the tasks of the research.

To start the process of creating a spatial routing for an array of points without reference to the road network, it is necessary to determine the initial data and set a task for constructing decision rules [1-6]. A solution is proposed and implemented in which an array of points and the connections between them are considered as an isomorphic undirected graph. The vertices of the graph correspond to a layer with point objects, and the edges correspond to a layer with linear objects.

Non-orientation indicates the presence of direct and reverse directions of movement between any pair of adjacent vertices. Each layer has its own table in the GIS Database. Each point feature has its own id, and the table for the line feature layer contains columns of attribute information about the id of the start and end points of each line. Therefore, we have complete information about the incidence of all elements of the graph with each other. The formation of the graph occurs as a result of determining the neighborhood for each vertex, therefore we can say that all private graphs with the beginning at the points of the array define each other and are uniquely connected - it follows that the property of isomorphism is inherent in the graph.

\section{Input data array}

Spatial routing is based on a number of geometric conditions, as well as conditions for interaction with surrounding objects. An intelligent agent must interact with an array of spatial data to fulfill decision rules [7]. Import of actual spatial data was made from the Open Street Map (OSM) server. The content and structure of the input data array for GIS intelligent agent located in the DBMS tables and presented in Table 1 and Figure 1.

Table 1. The content and structure of the DBMS tables of the input data array.

\begin{tabular}{|c|c|c|}
\hline $\begin{array}{c}\text { Table } \\
\text { name }\end{array}$ & \multicolumn{1}{|c|}{ Content } & \multicolumn{1}{c|}{ Table fields and data types } \\
\hline & An array of panoramic & $\begin{array}{l}\text { 1. ID (integer) - the number of point; } \\
\text { 2. Geom (geometry) - spatial coordinates of a }\end{array}$ \\
\hline
\end{tabular}




\begin{tabular}{|c|c|c|}
\hline Panoramas & $\begin{array}{l}\text { survey points from field } \\
\text { surveys }\end{array}$ & $\begin{array}{l}\text { given point; } \\
\text { 3. Region (integer) - affiliation of the point to } \\
\text { the survey area; }\end{array}$ \\
\hline Buildings & $\begin{array}{c}\text { An array of area objects } \\
\text { with the building tag } \\
\text { downloaded from the OSM } \\
\text { server }\end{array}$ & $\begin{array}{l}\text { 1. ID (integer) - the number of area object; } \\
\text { 2. Geom (geometry) - spatial coordinates of a } \\
\text { given area object; }\end{array}$ \\
\hline Highways & $\begin{array}{c}\text { An array of line objects } \\
\text { with the highway tag } \\
\text { downloaded from the OSM } \\
\text { server }\end{array}$ & $\begin{array}{l}\text { 1. ID (integer) - the number of line object; } \\
\text { 2. Geom (geometry) - spatial coordinates of a } \\
\text { given line object; }\end{array}$ \\
\hline Edges & $\begin{array}{l}\text { An array of linear objects } \\
\text { that connects the } \\
\text { panoramic survey points } \\
\text { from the Panoramas table. } \\
\text { The table is empty before } \\
\text { the intelligent agent starts }\end{array}$ & $\begin{array}{l}\text { 1. ID (integer) - the number of a given line } \\
\text { object (edge); } \\
\text { 2. Pano_1 (integer) - Panoramas.ID number } \\
\text { of the starting point of line object; } \\
\text { 3. Pano_2 (integer) - Panoramas.ID number } \\
\text { of the ending point of line object; } \\
\text { 4. Geom (geometry) - spatial coordinates of a } \\
\text { given line object; }\end{array}$ \\
\hline
\end{tabular}

Fig. 1. Mapping input dataset in QGIS.

\section{Geometric conditions}

Consider the sequence of geometric conditions for building a network.

1. The maximum distance between points (dist_max) does not exceed 100 meters. This restriction is due to the resolution of photographic systems and provides a physical access to observe an adjacent point of photography, while viewing this panoramic image. The distance value is a variable and is an input parameter for the intelligent agent.

2. The line (edge) connecting adjacent points of photography must not intersect any areal object with the tag 'building'. This condition ensures direct visibility between adjacent points of photography. 
3. The line (edge) connecting adjacent points of photography must not intersect any linear object with the tag 'highway'. This condition ensures the navigation zone within one city block, and also limits the possibility of building incorrect connections with the intersection of the roadway and violating the principle of logicality of spatial routing.

4. For each partial graph with an starting point $\mathrm{N}$ and neighborhood points satisfying the above conditions and belonging to an array of points in the table 'Panoramas', a necessary condition is the minimum central angle between any pair of edges (centr_ang_min) which is incident to the starting point $\mathrm{N}$ and to the corresponding points of the neighborhood. This fact allows to avoid cases in which the edges of a private graph are close to co-directed - it violates the principle of logicality and intuition of navigation. The executed studies allow us to conclude that a rectangular network is an ideal option. Having actual cartographic data, an approximate minimum central angle value of $40^{\circ}-60^{\circ}$ was obtained experimentally (the absolute value of the azimuth difference of any pair of edges of a private graph with a starting point $\mathrm{N}$ ). The value of the central angle is a variable, as well as the input parameter of the intelligent agent.

5. A private graph must not have more than 4 incident edges. This restriction is due to the convenience and intuition of user navigation and corresponds to the authors' idea of an ideal navigation network similar to a rectangular network. This restriction applies to any private graph.

6. If there are an excessive number of neighborhood points for the starting point $\mathrm{N}$ of any private graph, the incident edges should be sorted by increasing their length (geometry) and a restriction on the use of the first four edges from the resulting list should be established. Thus, the four most suitable points of the neighborhood will be determined.

7. If the value of the central angle between any pair of edges of the private graph which are incident to the starting point $\mathrm{N}$ and the corresponding points of the neighborhood is less than the angle in the set variable - then the priority is given to the edge with the shorter length (geometry), and the edge with the longer length (geometry) is removed from the private graph. In this case, the starting point $\mathrm{N}$ and the neighborhood point which is incident to the remote edge cease to be adjacent.

8. The navigation network should provide the ability to move the user in the forward and reverse direction between any pair of incident points of panoramic shooting. In this case, the navigation network should be considered as a graph with non-oriented (undirected) edges, and any incident points should be connected by lines in the forward and reverse directions.

\section{Building decision rules and their implementation by an intelligent GIS agent}

The developed intelligent agent is based on the implementation of crucial rules:

1. For each point of panoramic shooting (table Panoramas) we construct a buffer zone with a radius equal to the variable of maximum distance between points (buff_radius = dist max):

1.1. To all neighborhood points (table Panoramas), crossed by the buffer zone of this point, we build linear objects - we get a private oriented graph for this point.

1.2. Delete linear objects intersecting linear (table Highways) or areal objects (table Buildings) that have fallen into the buffer of this point. Neighborhood points satisfying 1, 2, and 3 geometric conditions are obtained.

2. For each private oriented graph, define all central angles (centr_ang) between all linear objects (edges). 
3. In each particular oriented graph, we make a selection of pairs of linear objects (edges), the central angle between which is less than the set minimum value (centr_ang $<$ centr_ang_min).

3.1. An object with a longer length is deleted from two conflicting. Thus, geometric conditions 4 and 7 are satisfied.

An example of selecting edges for points in a neighborhood of a private graph according to decision rules 1-3 is shown in Fig. 2.

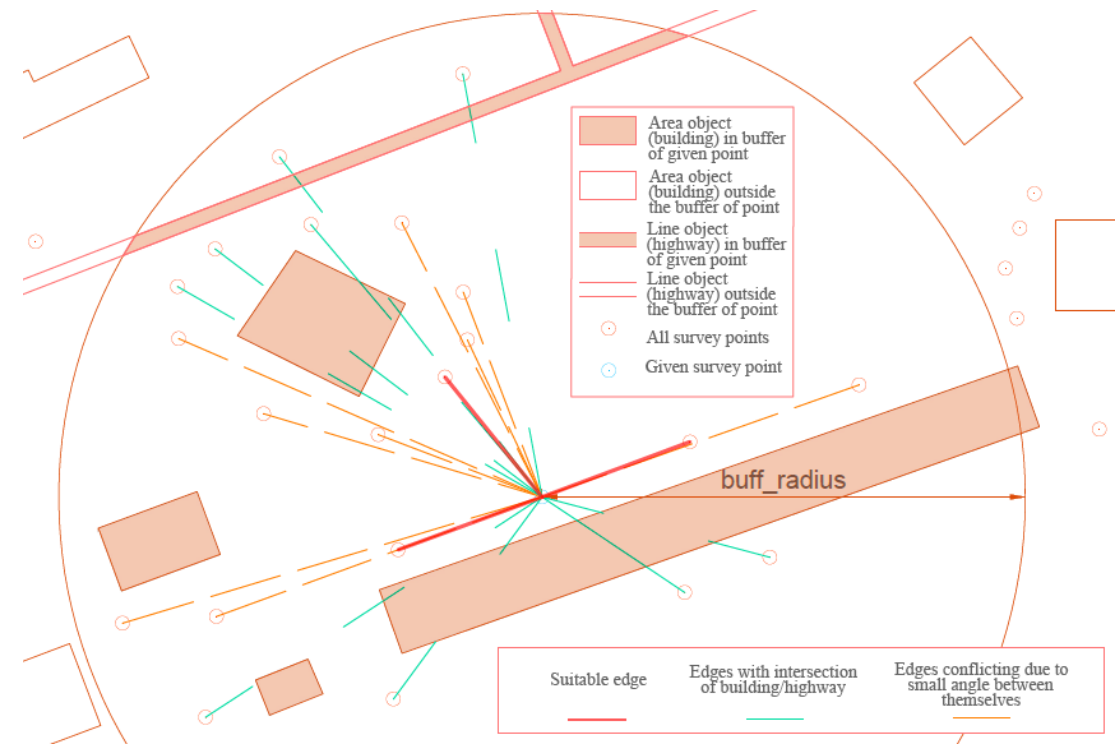

Fig. 2. An example of selecting edges for points in a neighborhood of a private graph according to decision rules 1-3.

4. Sort the edges in private graphs in increasing order and save the first 4 elements. Therefore, geometric conditions 5 and 6 are satisfied.

5. Complete the network with duplicating edges in the opposite direction. We obtain an undirected isomorphic graph according to geometric condition 8 .

Since private graphs were formed independently of each other, the isomorphism obtained from paragraph 5 leads to a violation of geometric conditions 4 and 5 .

6. Repeat paragraphs 3 and 3.1 for undirected private graphs with the removal of linear facilities (edges) in the forward and reverse directions.

It is necessary to filter linear facilities that divide the resulting rectangular network into groups of triangles.

7. In pairs of linear objects forming an acute angle at one common vertex and connected by another linear object through the remaining vertices, an linear object with a longer length is deleted.

A graphic illustration of the steps involved in implementing decision rules by a GIS intelligent agent is shown in Fig. 3. 


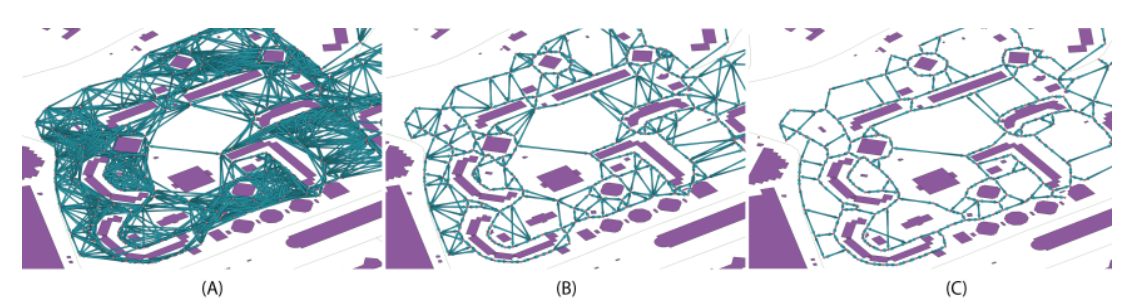

Fig. 3. Steps for implementing decision rules by an intelligent agent:

Stage A - Implementation of 1 decision rule;

Stage B - Implementation of decision rules 1-5 with the initial reduction of the graph to an isomorphic form;

Stage C - Implementation of decision rules 1-7.

\section{Conclusion}

The proposed method for constructing a spatial routing network provides movement within courtyards based on the construction of decision rules for a number of developed geometric conditions and network requirements. The research results allowed us to build a routing network based on real data for $1,500,000$ survey points, which completely covers Moscow city. Thus, the developed GIS intelligent agent has the ability to quickly generate a high-quality set of rational solutions, and it can be used for operational, reliable spatial routing inside courtyards without using information about the road network.

\section{References}

1. Hand, D. J., Data Mining, Encyclopedia of Environmetrics, 2 (2006)

2. Brown, D. G., Riolo, R., Robinson, D. T., North, M., \& Rand, W., Spatial process and data models: Toward integration of agent-based models and GIS, Journal of Geographical Systems, 7(1), 25-47 (2005)

3. Bell, M. G., Iida, Y., Transportation network analysis (1997)

4. Munkres, J., Topology (2014)

5. Fotheringham, S., Rogerson, P., Spatial analysis and GIS (2014)

6. Lee, J., A spatial access-oriented implementation of a 3-D GIS topological data model for urban entities, GeoInformatica, 8(3), 237-264 (2004)

7. Kwan, M. P., \& Hong, X. D, Network-based constraints-oriented choice set formation using GIS, Geographical Systems, 5, 148-153 (1998) 